\title{
On the Maximum Number of Period Annuli for Second Order Conservative Equations
}

\author{
Armands Gritsans and Inara Yermachenko \\ Daugavpils University, Institute of Life Sciences and Technology \\ Parādes str. $1^{a}$, LV-5400 Daugavpils, Latvia \\ E-mail(corresp.): inara.jermacenko@du.1v \\ E-mail: armands.gricans@du.lv
}

Received November 27, 2020; revised September 26, 2021; accepted September 27, 2021

\begin{abstract}
We consider a second order scalar conservative differential equation whose potential function is a Morse function with a finite number of critical points and is unbounded at infinity. We give an upper bound for the number of nonglobal nontrivial period annuli of the equation and prove that the upper bound obtained is sharp. We use tree theory in our considerations.
\end{abstract}

Keywords: conservative equation, Morse function, period annulus, binary tree.

AMS Subject Classification: 34C25; 05C90.

\section{Introduction}

In this paper, we consider a second order conservative differential equation

$$
x^{\prime \prime}=-U^{\prime}(x)
$$

where $U$ is a twice continuously differentiable function on the real line. The Equation (1.1) is equivalent to the planar system

$$
\left\{\begin{array}{l}
x^{\prime}=y, \\
y^{\prime}=-U^{\prime}(x) .
\end{array}\right.
$$

Following a mechanical interpretation of (1.1), see, for instance, [1], the function $U$ is called a potential energy function of (1.1).

Copyright (c) 2021 The Author(s). Published by Vilnius Gediminas Technical University This is an Open Access article distributed under the terms of the Creative Commons Attribution License (http://creativecommons.org/licenses/by/4.0/), which permits unrestricted use, distribution, and reproduction in any medium, provided the original author and source are credited. 
Definition 1. Suppose $U \in C^{2}(\mathbb{R}, \mathbb{R})$. A point $x_{0} \in \mathbb{R}$ is a critical point of $U$ if $U^{\prime}\left(x_{0}\right)=0$. A critical point $x_{0}$ of $U$ is nondegenerate, see [14], if $U^{\prime \prime}\left(x_{0}\right) \neq 0$. The function $U$ is said to be a Morse function, see [14], if all its critical points are nondegenerate.

In the present article, we consider functions $U$ satisfying the following conditions.

(A) The function $U$ is a Morse function with a finite number of critical points $\xi_{1}, \ldots, \xi_{n}$.

(B) The function $U$ satisfies one of the following conditions:

(B1) $n \geq 3$ and $\lim _{x \rightarrow \pm \infty} U(x)=+\infty$,

(B2) $n \geq 4, \lim _{x \rightarrow-\infty} U(x)=-\infty$, and $\lim _{x \rightarrow+\infty} U(x)=+\infty$,

(B3) $n \geq 4, \lim _{x \rightarrow-\infty} U(x)=+\infty$, and $\lim _{x \rightarrow+\infty} U(x)=-\infty$,

(B4) $n \geq 5$ and $\lim _{x \rightarrow \pm \infty} U(x)=-\infty$.

In what follows, we refer function $U$ satisfying (A) and (B) as a Morse potential.

If $U$ is a Morse potential, then $\xi_{i}(1 \leq i \leq n)$ are strict local minimum or maximum points for $U$. In what follows, we will assume that $\xi_{1}<\cdots<\xi_{n}$. In view of (B), a Morse potential $U$ has at least two strict local minimum points and at least one strict local maximum point; we denote by $n_{\min }$ and $n_{\max }$ the number of local minimum and maximum points of $U$, respectively. Then, $n_{\min }+n_{\max }=n$. The points $\left(\xi_{i}, 0\right), i \in\{1, \ldots, n\}$, are singular points of the planar system $(1.2)$ : a point $\left(\xi_{i}, 0\right)$ is a centre of $(1.2)$ if $U^{\prime \prime}\left(\xi_{i}\right)>0$ and a saddle of $(1.2)$ if $U^{\prime \prime}\left(\xi_{i}\right)<0$. The singular points of $(1.2)$ form a sequence $\left(\xi_{1}, 0\right), \ldots,\left(\xi_{n}, 0\right)$ of alternating centres and saddles on the horizontal axis of the phase plane of $(1.2)$.

We are looking for regions of the phase plane of (1.2) filled with nontrivial periodic orbits of (1.2). By region we mean a nonempty open connected subset of $\mathbb{R}^{2}$.

Definition 2. A maximal region covered with nontrivial periodic orbits of (1.2) is called a period annulus for (1.1). Maximality means that a period annulus is not contained in any other region covered with nontrivial periodic orbits of (1.2).

More generally, period annuli are similarly defined for autonomous planar systems as well. Some authors classify period annuli by the number of singular points enclosed by period annuli.

Definition 3. ( $[2,8,17])$ Let $(\xi, 0)$ be a singular point of $(1.2)$ and let $(\xi, 0)$ be a centre. The period annulus for (1.1) surrounding $(\xi, 0)$ is called a trivial period annulus or a central region for (1.1). A period annulus for (1.1) enclosing more than one singular point of (1.2) is called a nontrivial period annulus for (1.1). 
Definition 4. ( [22]) A period annulus for (1.1) enclosing all singular points of (1.2) is called a global period annulus for (1.1). Otherwise, it is called a nonglobal period annulus for (1.1).

If $U$ is a Morse potential, then $U$ has at least three critical points and thus a trivial period annulus for (1.1) is a nonglobal period annulus for (1.1). For the Equation (1.1) with a Morse potential $U$, by the phase plane analysis of (1.2), a global period annulus exists if and only if (B1) is fulfilled; moreover, it is unique.

The number of trivial, nontrivial, global, nonglobal, and nonglobal nontrivial period annuli for (1.1) are denoted by $N_{T}, N_{N T}, N_{G}, N_{N G}$, and $N_{N G, N T}$, respectively. By $N$ we denote the number of all period annuli for (1.1). In view of Definitions 3 and 4 ,

$$
N=N_{G}+N_{N G, N T}+N_{T}, \quad N_{T}=n_{\min }, \quad N_{N G, N T}=N_{N G}-N_{T} .
$$

In this paper, we are interested in finding a sharp upper bound for $N_{N G, N T}$.

We mention some references concerning period annuli for autonomous planar systems, in particular, for the systems (1.2). The period functions associated with central regions have been extensively studied, for instance, in $[10,11,16,21]$. Planar systems in which period annuli enclose limit cycles, or limit cycles enclose period annuli are studied, for instance, in $[2,5,6,17,22]$. In $[8,9]$, the authors study the maximum number of nontrivial period annuli for a conservative equation with a polynomial potential; the results obtained in $[8,9]$ are discussed in more detail at the end of this section.

Let us recall some concepts from the tree theory, see, for instance, $[18,19,20]$, that will be used in our article. A tree is a connected acyclic graph. A rooted tree is a tree that has one node designated as a root. Let $T$ be a rooted tree with at least two nodes and let $u_{0}$ be the root of $T$. Let $u$ be a node of $T$ that is different from $u_{0}$. There exists exactly one path in $T$ between $u_{0}$ and $u$, and thus there exists a unique node $v$ of $T$ such that the path contains the edge $\{v, u\}$. The node $v$ is called a parent node of $u$ and $u$ is called a child node of $v$. In a rooted tree, a leaf node is a node with no child nodes. A binary tree is a rooted tree in which all nodes have at most two child nodes.

The present article is organized as follows. In Section 2, we define critical segments for a Morse potential $U$ and explore their properties. In Section 3, we introduce the rooted tree $G(U)$ associated with a Morse potential $U$ in such a way that non-root nodes of $G(U)$ are critical segments for $U$. We show that the leaf nodes and the non-root non-leaf nodes of $G(U)$ represent the trivial and the nonglobal nontrivial period annuli for (1.1), respectively. Let $\alpha$ be a real number. Then, $\lfloor\alpha\rfloor:=\max \{k \in \mathbb{Z}: k \leq \alpha\}$ is the floor of $\alpha$ and $\lceil\alpha\rceil:=\min \{k \in \mathbb{Z}: k \geq \alpha\}$ is the ceiling of $\alpha$. In Section 4 , we prove the first main theorem of our article.

Theorem 1. Let $U$ be a Morse potential with $n$ critical points. The number of nonglobal nontrivial period annuli for (1.1) satisfies the following inequality:

$$
N_{N G, N T} \leq\left\lfloor\frac{n}{2}\right\rfloor-1
$$


In Section 5, at first, we prove that, for a Morse potential $U$, providing the inequality (1.4) becomes equality, the associated rooted tree $G(U)$ is a binary tree with additional structure. Then, we prove the second main theorem of our article.

Theorem 2. The following statements are valid.

(1) For every odd integer $n(n \geq 3)$, there is a Morse potential $U$ with $n$ critical points such that $U$ satisfies (B1) and ensures equality in (1.4).

(2) For every even integer $n(n \geq 4)$, there is a Morse potential $U$ with $n$ critical points such that $U$ satisfies (B2) and ensures equality in (1.4).

(3) For every even integer $n(n \geq 4)$, there is a Morse potential $U$ with $n$ critical points such that $U$ satisfies (B3) and ensures equality in (1.4).

(4) For every odd integer $n(n \geq 5)$, there is a Morse potential $U$ with $n$ critical points such that $U$ satisfies (B4) and ensures equality in (1.4).

The main theorems in our article provide a wide generalization of the results obtained in $[8,9]$. In the last two references, the authors explore the Equation (1.1) for a real polynomial $U$ of degree $n+1$ ( $n$ is odd and $n \geq 5$ ) such that the function $U$ has $n$ nondegenerate critical points, and the leading coefficient of $U$ is negative. Hence, $U$ satisfies (A) and (B4) and thus $n_{\max }=n_{\min }+1$. It follows from Theorem 1 that $N_{N G, N T} \leq\left\lfloor\frac{n}{2}\right\rfloor-1=\left\lfloor\frac{2 n_{\max }-1}{2}\right\rfloor-1=n_{\max }-2$. The condition (B4) implies $N_{N G, N T}=N_{N T}$. Hence, we obtain the inequality $N_{N T} \leq n_{\max }-2$, which was proved in [9]. In [8,9], the authors proved the sharpness of the last inequality.

It is worth mentioning that graph theory is often used to study Morse functions, for example, Reeb graphs are important for exploring Morse functions over manifolds, see, for instance, $[4,7,12,13]$ and references therein. Reeb graphs are used also in computer graphics, see [4]. In [12], conditions are indicated under which Reeb graph is a tree. In $[7,13]$, the authors provide the upper bound for the number of cycles in Reeb graphs and prove that the upper bound is sharp. (Compare with the main theorems in this article.) In our opinion, this article contributes to the application of graph theory to the study of Morse functions and differential equations.

\section{Critical segments for Morse potentials}

Let $U$ be a Morse potential. For every $c \in \mathbb{R}$, by Rolle's theorem, the preimage $U^{-1}(c):=\{x \in \mathbb{R}: U(x)=c\}$ of the point $c$ under $U$ is a finite set.

Definition 5. Let $a$ and $b$ be real numbers. Suppose $a<b$ and $U(a)=U(b)=$ : $M$. Let $a$ or $b$ (may be both) be a local maximum point of $U$. The closed line segment $\mathcal{E}=[(a, M),(b, M)] \subset \mathbb{R}^{2}$ is a critical segment for $U$, shortly CS, if $U(x)<M$ for every $x \in(a, b)$. The value $M$ is an energy level of $\mathcal{E}$. Let $\xi$ be a local maximum point of $U$. We say the point $(\xi, U(\xi))$ generates a CS $\mathcal{E}$ for $U$ if $(\xi, U(\xi)) \in \mathcal{E}$. 
Definition 6. Let $\mathcal{E}_{1}=\left[\left(a_{1}, M_{1}\right),\left(b_{1}, M_{1}\right)\right]$ and $\mathcal{E}_{2}=\left[\left(a_{2}, M_{2}\right),\left(b_{2}, M_{2}\right)\right]$ be two CS for $U$. We say $\mathcal{E}_{1}$ is a parent CS of $\mathcal{E}_{2}$ and $\mathcal{E}_{2}$ is a child CS of $\mathcal{E}_{1}$ if $\left[a_{2}, b_{2}\right] \subset\left(a_{1}, b_{1}\right)$, the inequality $M_{2}<M_{1}$ holds, and $U(\eta) \leq M_{2}$ for every local maximum point $\eta$ of $U$ in the interval $\left(a_{1}, b_{1}\right)$.

Remark 1. Let $E_{0}=\left[\left(a_{0}, M_{0}\right),\left(b_{0}, M_{0}\right)\right], E_{1}=\left[\left(a_{1}, M_{1}\right),\left(b_{1}, M_{1}\right)\right]$, and $E_{2}=$ $\left[\left(a_{2}, M_{2}\right),\left(b_{2}, M_{2}\right)\right]$ be three CS for $U$. Suppose that $E_{0}$ is a parent CS for $E_{1}$ and $E_{1}$ is a parent CS of $E_{2}$, that is, $E_{0}$ is a grandparent CS of $E_{2}$. Then, $\left[a_{2}, b_{2}\right] \subset\left[a_{1}, b_{1}\right] \subset\left(a_{0}, b_{0}\right)$ and $M_{2}<M_{1}<M_{0}$. Since $E_{1}$ is a CS for $U$, we see that $a_{1}$ or $b_{1}$ (may be both) is a local maximum point of $U$ and $U\left(a_{1}\right)=U\left(b_{1}\right)=$ $M_{1}$. Assume that $a_{1}$ is a local maximum point of $U$. Hence, there exists a local maximum point $a_{1}$ of $U$ in the interval $\left(a_{0}, b_{0}\right)$ such that $U\left(a_{1}\right)>M_{2}$ and thus the grandparent CS $E_{0}$ of $E_{2}$ is not a parent CS of $E_{2}$.

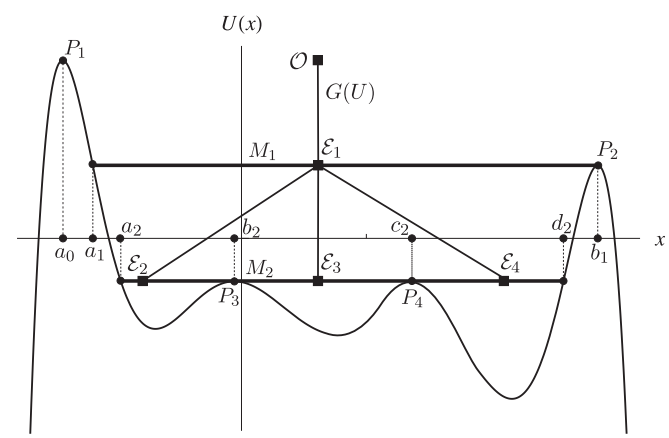

Figure 1. A Morse potential $U$ has seven critical points and satisfies (B4). The function $U$ has four CS $\mathcal{E}_{1}=\left[\left(a_{1}, M_{1}\right),\left(b_{1}, M_{1}\right)\right], \mathcal{E}_{2}=\left[\left(a_{2}, M_{2}\right),\left(b_{2}, M_{2}\right)\right], \mathcal{E}_{3}=\left[\left(b_{2}, M_{2}\right),\left(c_{2}, M_{2}\right)\right]$, and $\mathcal{E}_{4}=\left[\left(c_{2}, M_{2}\right),\left(d_{2}, M_{2}\right)\right]$, which are nodes of the associated rooted tree $G(U)$.

Example 1. In Figure 1, the points $a_{0}, b_{1}, b_{2}$, and $c_{2}$ are local maximum points of $U$. The point $P_{1}=\left(a_{0}, U\left(a_{0}\right)\right)$ does not generate any CS for $U$, the point $P_{2}=\left(b_{1}, U\left(b_{1}\right)\right)$ generates exactly one CS $\mathcal{E}_{1}$ for $U$, the point $P_{3}=\left(b_{2}, U\left(b_{2}\right)\right)$ generates exactly two CS $\mathcal{E}_{2}$ and $\mathcal{E}_{3}$ for $U$, and the point $P_{4}=\left(c_{2}, U\left(c_{2}\right)\right)$ generates exactly two $\mathrm{CS} \mathcal{E}_{3}$ and $\mathcal{E}_{4}$ for $U$. In the same figure, $\mathcal{E}_{1}$ is a parent CS of $\mathcal{E}_{2}, \mathcal{E}_{3}$, and $\mathcal{E}_{4}$ and the last three CS are child CS of $\mathcal{E}_{1}$.

In the next proposition, we collect properties of CS.

Proposition 1. Let $U$ be a Morse potential.

(a) Let $\xi$ be a local maximum point of $U$. The point $(\xi, U(\xi))$ generates at most two CS for $U$. Any CS for $U$ is generated by either one or both of its endpoints.

(b) The function $U$ has at least two $C S$ and the number of $C S$ for $U$ is finite.

(c) $A C S \mathcal{E}_{1}=\left[\left(a_{1}, M_{1}\right),\left(b_{1}, M_{1}\right)\right]$ for $U$ has a child $C S$ if and only if the interval $\left(a_{1}, b_{1}\right)$ contains at least one local maximum point of $U$. If a 
$C S \mathcal{E}_{1}$ has a child $C S$, then $\mathcal{E}_{1}$ has at least two child CS. A union of all child $C S$ for $\mathcal{E}_{1}$ is a closed line segment $\left[\left(a_{2}, M_{2}\right),\left(b_{2}, M_{2}\right)\right]$, where $\left[a_{2}, b_{2}\right] \subset\left(a_{1}, b_{1}\right)$.

(d) $A C S \mathcal{E}_{2}=\left[\left(a_{2}, M_{2}\right),\left(b_{2}, M_{2}\right)\right]$ for $U$ has a parent $C S$ if and only if there exists a local maximum $M_{1}$ of $U$ such that $M_{1}>M_{2}$ and both the sets $U^{-1}\left(M_{1}\right) \cap\left(-\infty, a_{2}\right)$ and $U^{-1}\left(M_{1}\right) \cap\left(b_{2},+\infty\right)$ are nonempty.

(e) Every CS for $U$ has at most one parent CS.

(f) Let $\mathcal{E}_{1}=\left[\left(a_{1}, M_{1}\right),\left(b_{1}, M_{1}\right)\right]$ and $\mathcal{E}_{2}=\left[\left(a_{2}, M_{2}\right),\left(b_{2}, M_{2}\right)\right]$ be two different $C S$ for $U$ such that $M_{2} \leq M_{1}$. Then, either the intervals $\left(a_{1}, b_{1}\right)$ and $\left(a_{2}, b_{2}\right)$ do not intersect or $\left[a_{2}, b_{2}\right] \subset\left(a_{1}, b_{1}\right)$.

Proof. (a) Let $\xi$ be a local maximum point of $U$ and let $M$ stand for $U(\xi)$. Let $\mathcal{E}_{1}$ and $\mathcal{E}_{2}$ be two different CS for $U$ with the same energy level $M$. It follows from Definition 5 that either $\mathcal{E}_{1}$ and $\mathcal{E}_{2}$ do not intersect or the intersection of $\mathcal{E}_{1}$ and $\mathcal{E}_{2}$ is a singleton set consisting of the common endpoint of $\mathcal{E}_{1}$ and $\mathcal{E}_{2}$. Consequently, the point $(\xi, U(\xi))$ can belong to at most two CS for $U$ with the energy level $M$. In view of Definition 5 , the point $(\xi, U(\xi))$ generates at most two CS for $U$. If $\mathcal{E}=[(a, M),(b, M)]$ is a CS for $U$, then either the point $(a, M)$ generates $\mathcal{E}$ and the point $(b, M)$ does not generate $\mathcal{E}$, the point $(a, M)$ does not generate $\mathcal{E}$ and the point $(b, M)$ generates $\mathcal{E}$, or both the points $(a, M)$ and $(b, M)$ generate $\mathcal{E}$.

(b) It follows from (A) and (B) that there exist two local minimum points $\eta_{1}$ and $\eta_{2}$ of $U$ such that $\eta_{1}<\eta_{2}$ and the interval $\left(\eta_{1}, \eta_{2}\right)$ does not contain local minimum points of $U$. Then, there exists a unique local maximum point $\xi$ of $U$ in the interval $\left(\eta_{1}, \eta_{2}\right)$. Let $M$ stand for $U(\xi)$. We provide the proof only for the case when (B2) is valid. Since $\lim _{x \rightarrow-\infty} U(x)=-\infty$, we conclude that the interval $(-\infty, \xi)$ contains local maximum points of $U$. Let $c_{1}$ be the local maximum point of $U$ closest to $\xi$ on the left. Consider $M_{1}:=\min \left\{U\left(c_{1}\right), M\right\}$. By the intermediate value theorem, there exist unique elements $a_{1}$ and $b_{1}$ of $U^{-1}\left(M_{1}\right)$ in the intervals $\left[c_{1}, \eta_{1}\right)$ and $\left(\eta_{1}, \xi\right]$, respectively. Then, $\mathcal{E}_{1}=\left[\left(a_{1}, M_{1}\right),\left(b_{1}, M_{1}\right)\right]$ is a CS for $U$. Since $\lim _{x \rightarrow+\infty} U(x)=+\infty$, we see that the set $U^{-1}(M) \cap(\xi,+\infty)$ is nonempty. Let $b_{2}$ be the element of $U^{-1}(M)$ closest to $\xi$ on the right. Then, $\mathcal{E}_{2}=\left[(\xi, M),\left(b_{2}, M\right)\right]$ is a CS for $U$. Since $a_{1}<b_{1} \leq \xi<b_{2}$, we see that $\mathcal{E}_{1} \neq \mathcal{E}_{2}$. Hence, $U$ has at least two CS. It follows from (a) that every local local maximum point of $U$ generates at most two CS for $U$. Since $U$ has a finite number of local maximum points, we conclude that the number of CS for $U$ is finite.

(c) If a CS $\mathcal{E}_{1}=\left[\left(a_{1}, M_{1}\right),\left(b_{1}, M_{1}\right)\right]$ has a child CS $\mathcal{E}_{2}=\left[\left(a_{2}, M_{2}\right),\left(b_{2}, M_{2}\right)\right]$, then $a_{2}$ or $b_{2}$ is a local maximum point of $U$ in the interval $\left(a_{1}, b_{1}\right)$.

Let $\mathcal{E}_{1}=\left[\left(a_{1}, M_{1}\right),\left(b_{1}, M_{1}\right)\right]$ be a CS for $U$. Suppose that $U$ has a local maximum point in the interval $\left(a_{1}, b_{1}\right)$. Consider all local maximum points $\eta_{1}, \ldots, \eta_{r}(r \geq 1)$ in the interval $\left(a_{1}, b_{1}\right)$ and find $M_{2}=\max \left\{U\left(\eta_{1}\right), \ldots, U\left(\eta_{r}\right)\right\}$. Then, $U\left(\eta_{p}\right) \leq M_{2}$ for every $p \in\{1, \ldots, r\}$. Since $\mathcal{E}_{1}=\left[\left(a_{1}, M_{1}\right),\left(b_{1}, M_{1}\right)\right]$ is a $\mathrm{CS}$, we see that $M_{2}<M_{1}$. Consider all local maximum points $c_{i}(1 \leq i \leq m)$ 
of $U$, where $1 \leq m \leq r$, such that $c_{i} \in\left(a_{1}, b_{1}\right)$ and $U\left(c_{i}\right)=M_{2}$ for every $i \in\{1, \ldots, m\}$. Find $c_{\text {min }}:=\min _{1 \leq i \leq m}\left\{c_{i}\right\}$ and $c_{\text {max }}:=\max _{1 \leq i \leq m}\left\{c_{i}\right\}$. By the intermediate value theorem, there exist unique elements $c_{0}$ and $c_{m+1}$ of $U^{-1}\left(M_{2}\right)$ in the intervals $\left(a_{1}, c_{\min }\right)$ and $\left(c_{\max }, b_{1}\right)$, respectively. If $m=1$, then $c_{\min }=$ $c_{1}=c_{\max }$ and hence $\mathcal{E}_{1}$ has exactly two child CS $\mathcal{E}_{0}^{\prime}=\left[\left(c_{0}, M_{2}\right),\left(c_{1}, M_{2}\right)\right]$ and $\mathcal{E}_{1}^{\prime}=\left[\left(c_{1}, M_{2}\right),\left(c_{2}, M_{2}\right)\right]$. Suppose $m \geq 2$. Without loss of generality, we can assume that $c_{1}<\cdots<c_{m}$. Then, $\mathcal{E}_{0}^{\prime}=\left[\left(c_{0}, M_{2}\right),\left(c_{1}, M_{2}\right)\right]$, $\mathcal{E}_{1}^{\prime}=\left[\left(c_{1}, M_{2}\right),\left(c_{2}, M_{2}\right)\right], \ldots, \mathcal{E}_{m}^{\prime}=\left[\left(c_{m}, M_{2}\right),\left(c_{m+1}, M_{2}\right)\right]$ is a list of all child $\mathrm{CS}$ of $\mathcal{E}_{1}$.

If a CS $\mathcal{E}_{1}$ has a child CS, then $U$ has a local maximum point in the interval $\left(a_{1}, b_{1}\right)$. It follows from the above considerations that $\mathcal{E}_{1}$ has at least two child CS and a union of CS $\mathcal{E}_{i}^{\prime}(0 \leq i \leq m)$ is equal to the closed line segment $\left[\left(a_{2}, M_{2}\right),\left(b_{2}, M_{2}\right)\right]$, where $a_{2}=c_{0}$ and $b_{2}=c_{m+1}$, and $\left[a_{2}, b_{2}\right] \subset\left(a_{1}, b_{1}\right)$.

(d) The proof is similar to the one of (c).

(e) Let $\mathcal{E}_{2}=\left[\left(a_{2}, M_{2}\right),\left(b_{2}, M_{2}\right)\right]$ be a CS for $U$. Suppose that $\mathcal{E}_{1}^{\prime}=$ $\left[\left(a_{1}^{\prime}, M_{1}^{\prime}\right),\left(b_{1}^{\prime}, M_{1}^{\prime}\right)\right]$ and $\mathcal{E}_{1}^{\prime \prime}=\left[\left(a_{1}^{\prime \prime}, M_{1}^{\prime \prime}\right),\left(b_{1}^{\prime \prime}, M_{1}^{\prime \prime}\right)\right]$ are parent CS of $\mathcal{E}_{2}$. Then, the following inequalities hold: $M_{2}<M_{1}^{\prime}, M_{2}<M_{1}^{\prime \prime}, a_{1}^{\prime}<a_{2}<b_{2}<b_{1}^{\prime}$, and $a_{1}^{\prime \prime}<a_{2}<b_{2}<b_{1}^{\prime \prime}$. Let us prove that $M_{1}^{\prime}=M_{1}^{\prime \prime}$. Suppose to the contrary that $M_{1}^{\prime} \neq M_{1}^{\prime \prime}$. Without loss of generality, we can assume that $M_{1}^{\prime}<M_{1}^{\prime \prime}$. Since $\mathcal{E}_{1}^{\prime}$ is a CS, we conclude that $a_{1}^{\prime}$ or $b_{1}^{\prime}$ is a local maximum point of $U$. Suppose that $a_{1}^{\prime}$ is a local maximum point of $U$. If $a_{1}^{\prime \prime}<a_{1}^{\prime}$, then the interval $\left(a_{1}^{\prime \prime}, b_{1}^{\prime \prime}\right)$ contains a local maximum point $a_{1}^{\prime}$ of $U$ and $U\left(a_{1}^{\prime}\right)=M_{1}^{\prime}>M_{2}$, which contradicts to $\mathcal{E}_{1}^{\prime \prime}$ is a parent CS of $\mathcal{E}_{2}$. If $a_{1}^{\prime \prime}=a_{1}^{\prime}$, then $M_{1}^{\prime}=U\left(a_{1}^{\prime}\right)=U\left(a_{1}^{\prime \prime}\right)=M_{1}^{\prime \prime}$, which contradicts to the hypothesis. If $a_{1}^{\prime \prime}>a_{1}^{\prime}$, then the interval $\left(a_{1}^{\prime}, b_{1}^{\prime}\right)$ contains a point $a_{1}^{\prime \prime}$ such that $U\left(a_{1}^{\prime \prime}\right)=M_{1}^{\prime \prime}>M_{1}^{\prime}$, which contradicts to $\mathcal{E}_{1}^{\prime}$ is a CS. The case when $b_{1}^{\prime}$ is a local maximum point of $U$ is considered similarly. Therefore, $M_{1}^{\prime}=M_{1}^{\prime \prime}=: M_{1}$. Let us prove that $a_{1}^{\prime}=a_{1}^{\prime \prime}$ and $b_{1}^{\prime}=b_{1}^{\prime \prime}$. Suppose to the contrary that $a_{1}^{\prime} \neq a_{1}^{\prime \prime}$ or $b_{1}^{\prime} \neq b_{1}^{\prime \prime}$. Suppose $a_{1}^{\prime} \neq a_{1}^{\prime \prime}$. If $a_{1}^{\prime}<a_{1}^{\prime \prime}$, then $a_{1}^{\prime \prime} \in\left(a_{1}^{\prime}, b_{1}^{\prime}\right)$ and $U\left(a_{1}^{\prime \prime}\right)=M_{1}$, which contradicts to $\mathcal{E}_{1}^{\prime}$ is a CS. If $a_{1}^{\prime}>a_{1}^{\prime \prime}$, then $a_{1}^{\prime} \in\left(a_{1}^{\prime \prime}, b_{1}^{\prime \prime}\right)$ and $U\left(a_{1}^{\prime}\right)=M_{1}$, which contradicts to $\mathcal{E}_{1}^{\prime \prime}$ is a CS. The case $b_{1}^{\prime} \neq b_{1}^{\prime \prime}$ also leads to a contradiction. Thereby, $a_{1}^{\prime}=a_{1}^{\prime \prime}$ and $b_{1}^{\prime}=b_{1}^{\prime \prime}$ and thus $\mathcal{E}_{1}^{\prime}=\mathcal{E}_{1}^{\prime \prime}$.

(f) The proof follows from Definition 5 and the proof of (a).

\section{Rooted trees associated with Morse potentials}

Let $U$ be a Morse potential. It follows from Proposition 1(b) that $U$ has at least two CS and the number of CS for $U$ is finite. Let $\theta$ be a real number and let $M_{0}$ be a real number greater than the maximum energy level of all CS for $U$. Let $V(U)$ be a set of nodes consisting of all CS for $U$ and the point $\mathcal{O}:=\left(\theta, M_{0}\right)$. Hence, the set $V(U)$ is finite and it contains at least three elements. In view of Proposition 1(e), for an arbitrary CS $\mathcal{E}$ for $U$, either $\mathcal{E}$ has a unique parent CS or $\mathcal{E}$ has no parent CS. Let $E(U)$ be a set of edges consisting of all pairs $\left\{\mathcal{E}, \mathcal{E}^{\prime}\right\}$ if $\mathcal{E}$ has a parent CS $\mathcal{E}^{\prime}$ and all pairs $\{\mathcal{E}, \mathcal{O}\}$ if $\mathcal{E}$ has no parent CS. Hence, if $u$ and $v$ are CS for $U$ and $\{u, v\} \in E(U)$, then either $u$ is a parent CS of $v$ or $v$ is a parent $\operatorname{CS}$ of $u$. 
Definition 7. Let $U$ be a Morse potential. The graph $G(U):=(V(U), E(U))$ is said to be the associated graph with $U$.

Denote by $N_{C S}$ the number of critical segments for $U$. We conclude that the graph $G(U)$ has $N_{C S}+1$ nodes and $N_{C S}$ edges. We designate the node $\mathcal{O}$ as a root of $G(U)$. Hence, $G(U)$ is a rooted graph with the root $\mathcal{O}$.

Proposition 2. For a Morse potential $U$, the graph $G(U)$ is a rooted tree.

Proof. Let us prove that $G(U)$ does not contain cycles. Suppose to the contrary that $G(U)$ contains a cycle $C=\left(u_{1}, u_{2}, \ldots, u_{k}, u_{k+1}\right)$, where $k \geq 3$ and $u_{k+1}=u_{1}$. Then, the nodes $u_{1}, u_{2}, \ldots, u_{k}$ are different and the cycle $C$ has the edges $\left\{u_{1}, u_{2}\right\},\left\{u_{2}, u_{3}\right\}, \ldots,\left\{u_{k}, u_{k+1}\right\}$.

Suppose $\mathcal{O} \notin\left\{u_{1}, \ldots, u_{k}\right\}$; then, the nodes $u_{1}, \ldots, u_{k}$ are CS for $U$. For every $j \in\{1, \ldots, k\}$, let $M_{j}$ be an energy level for $u_{j}$. Since $\left\{u_{1}, u_{2}\right\} \in E(U)$, then either $u_{1}$ is a parent CS of $u_{2}$ or $u_{2}$ is a parent CS of $u_{1}$. Without loss of generality, we can assume that $u_{1}$ is a parent CS of $u_{2}$. Hence, $M_{1}>M_{2}$. For the edge $\left\{u_{2}, u_{3}\right\}$, it follows from Proposition $1(\mathrm{e})$ that $u_{2}$ is a parent CS of $u_{3}$ and thus $M_{2}>M_{3}$. Continuing, for the edge $\left\{u_{k}, u_{k+1}\right\}$, it follows from Proposition 1(e) that $u_{k}$ is a parent CS of $u_{k+1}$. Since $u_{k+1}=u_{1}$, we see that $M_{k}>M_{1}$. Therefore, $M_{1}>M_{2}>\cdots>M_{k}>M_{1}$ which leads to a contradiction.

Suppose $\mathcal{O} \in\left\{u_{1}, u_{2}, \ldots, u_{k}\right\}$. Without loss of generality, we can assume that $\mathcal{O}=u_{1}$. Then, the cycle $C$ contains the edge $\left\{\mathcal{O}, u_{2}\right\}$ and thus $u_{2}$ has no parent CS. For the edge $\left\{u_{2}, u_{3}\right\}$, it follows from Proposition $1(\mathrm{e})$ that $u_{2}$ is a parent CS of $u_{3}$. Continuing, for the edge $\left\{u_{k-1}, u_{k}\right\}$, it follows from Proposition $1(\mathrm{e})$ that $u_{k-1}$ is a parent CS of $u_{k}$. Finally, for the edge $\left\{u_{k}, u_{k+1}\right\}=\left\{u_{k}, \mathcal{O}\right\}, u_{k}$ has no parent CS. The contradiction obtained proves that $G(U)$ does not contain cycles. Since $G(U)$ has $N_{C S}+1$ nodes and $N_{C S}$ edges, it follows from [20, Theorem 2.4.1.] that $G(U)$ is a tree.

In Figure 1, a Morse potential $U$ and the associated rooted tree $G(U)$ are depicted. It follows from Proposition 1, that the number $N_{C S}$ of CS for (1.1) is finite and $N_{C S} \geq 2$. To every CS $\mathcal{E}$ for $U$, we associate a period annulus $\mathcal{A}(\mathcal{E})$ as follows.

Suppose a CS $\mathcal{E}_{1}=\left[\left(a_{1}, M_{1}\right),\left(b_{1}, M_{1}\right)\right]$ has a child CS. It follows from Proposition 1(c) that $\mathcal{E}_{1}$ has at least two child CS and a union of all child CS for $\mathcal{E}_{1}$ is a closed line segment $\left[\left(a_{2}, M_{2}\right),\left(b_{2}, M_{2}\right)\right]$, where $\left[a_{2}, b_{2}\right] \subset\left(a_{1}, b_{1}\right)$. Taking into account [1], solutions of (1.2) that start at the points $(\alpha, 0)$, where $\alpha \in$ $\left(a_{1}, a_{2}\right)$, or, equivalently solutions of $(1.2)$ that start at the points $(\beta, 0)$, where $\beta \in\left(b_{2}, b_{1}\right)$, are periodic and form the nonglobal nontrivial period annulus $\mathcal{A}\left(\mathcal{E}_{1}\right)$ of (1.1) associated with $\mathcal{E}_{1}$.

Suppose a CS $\mathcal{E}_{1}=\left[\left(a_{1}, M_{1}\right),\left(b_{1}, M_{1}\right)\right]$ has no child CS. In view of Proposition $1(\mathrm{c})$, the interval $\left(a_{1}, b_{1}\right)$ contains a unique critical point $\xi$ of $U$, which is a local minimum point. Hence, $(\xi, 0)$ is a centre of (1.2). Taking into account [1], solutions of (1.2) that start at the points $(\alpha, 0)$, where $\alpha \in\left(a_{1}, \xi\right)$, or, equivalently solutions of (1.2) that start at the points $(\beta, 0)$, where $\beta \in\left(\xi, b_{1}\right)$, are periodic and form the trivial period annulus $\mathcal{A}\left(\mathcal{E}_{1}\right)$ of (1.1) associated with $\mathcal{E}_{1}$. 
In view of (B), a trivial period annulus of (1.1) is a nonglobal period annulus of (1.1). Thereby, the correspondence $\mathcal{E} \mapsto \mathcal{A}(\mathcal{E})$ defines a mapping $\mathcal{A}$ from the set $\mathcal{C}$ of all $\mathrm{CS}$ for $U$ to the set $\mathcal{P}_{N G}$ of all nonglobal period annuli for (1.1). Using the phase plane analysis of the planar system (1.2) and taking into account Proposition 1, we deduce that $\left(\mathrm{I}^{\prime}\right)$ the mapping $\mathcal{A}: \mathcal{C} \mapsto \mathcal{P}_{N G}$ is one-to-one and thus

$$
N_{C S}=N_{N G}
$$

(II') under the mapping $\mathcal{A}: \mathcal{C} \mapsto \mathcal{P}_{N G}$, the CS for $U$ that have child CS are in one-to-one correspondence with the nonglobal nontrivial period annuli for (1.1); (III') under the mapping $\mathcal{A}: \mathcal{C} \mapsto \mathcal{P}_{N G}$, the CS for $U$ that have no child $\mathrm{CS}$ are in one-to-one correspondence with the trivial period annuli for (1.1); therefore, combining (1.3) and (3.1), we obtain

$$
N_{N G, N T}=N_{C S}-n_{\min } .
$$

Taking into account Propositions 1 and 2 and the assertions $\left(\mathrm{I}^{\prime}\right)-\left(\mathrm{III}^{\prime}\right)$, we conclude that the following proposition holds.

Proposition 3. Let $U$ be a Morse potential. The following statements are valid:

(I) the nonglobal period annuli for (1.1) are in one-to-one correspondence with the non-root nodes of $G(U)$;

(II) the nonglobal nontrivial period annuli for (1.1) are in one-to-one correspondence with the non-root non-leaf nodes of $G(U)$;

(III) the trivial period annuli for (1.1) are in one-to-one correspondence with the leaf nodes of $G(U)$.

\section{Proof of the first main theorem}

Let $X$ and $Y$ be two nonempty sets. Denote by $\mathcal{P}(Y)$ the power set of $Y$. A multivalued mapping $\varphi$ from the set $X$ to the set $Y$ is a mapping from the set $X$ to the set $\mathcal{P}(Y)$ such that $\varphi(x)$ is a nonempty subset of $Y$ for every $x \in X$, see [3]. The multivalued mapping $\varphi$ from $X$ to $Y$ is denoted by $\varphi: X \rightrightarrows Y$. Let $B$ be a nonempty subset of $Y$. Consider the set $\varphi^{-}(B):=\{x \in X: \varphi(x) \cap B \neq \emptyset\}$, see [3, p. 24]. If $B$ is a singleton set, say $B=\{b\}$, then $\varphi^{-}(B)$ is denoted by $\varphi^{-}(b)$; hence $\varphi^{-}(b)=\{x \in X: b \in \varphi(x)\}$. The cardinality of a finite set $S$ is denoted by $|S|$. Assume that $X$ and $Y$ are nonempty finite sets and $Y=\left\{P_{1}, \ldots, P_{q}\right\}$, where $q \geq 1$. For a multivalued mapping $\varphi: X \rightrightarrows Y$,

$$
X=\varphi^{-}\left(P_{1}\right) \cup \cdots \cup \varphi^{-}\left(P_{q}\right) .
$$

Suppose that a Morse potential $U$ has local maximum points $\eta_{i}(1 \leq i \leq$ $\left.n_{\max }\right)$. Consider the points $P_{i}:=\left(\eta_{i}, U\left(\eta_{i}\right)\right)\left(1 \leq i \leq n_{\max }\right)$ and the set $Y:=\left\{P_{1}, \ldots, P_{n_{\max }}\right\}$. In accordance with Proposition 1(b), the function $U$ has at least two CS and the number of CS for $U$ is finite. Consider the set $\mathcal{C}=\left\{\mathcal{E}_{1}, \ldots, \mathcal{E}_{N_{C S}}\right\}$ of all CS for $U$. Let us define a multivalued mapping 
$\varphi: \mathcal{C} \rightrightarrows Y$ as follows: for every $j \in\left\{1, \ldots, N_{C S}\right\}$, the value $\varphi\left(\mathcal{E}_{j}\right)$ is a subset of $Y$ consisting of the points that generate $\mathcal{E}_{j}$. On account of Proposition 1(a), the cardinality $\left|\varphi\left(\mathcal{E}_{j}\right)\right| \in\{1,2\}$ for every $j \in\left\{1, \ldots, N_{C S}\right\}$. For every $i \in$ $\left\{1, \ldots, n_{\max }\right\}$, the set $\varphi^{-}\left(P_{i}\right)$ consists of the CS for $U$ generated by the point $P_{i}$. Then, $k_{i}:=\left|\varphi^{-}\left(P_{i}\right)\right|\left(1 \leq i \leq n_{\max }\right)$ is the number of the CS for $U$ generated by the point $P_{i}$. It follows from Proposition 1 (a) that $k_{i} \in\{0,1,2\}$ for every $i \in\left\{1, \ldots, n_{\max }\right\}$. Let $J_{1}, \ldots, J_{t}(t \geq 1)$ be a partition of the set $\left\{1, \ldots, n_{\max }\right\}$, where among the sets $J_{1}, \ldots, J_{t}(t \geq 1)$ there may be empty sets; see [15, p. 130]. Since $|\mathcal{C}|=N_{C S}$, it follows from (4.1) that

$$
N_{C S}=\left|\varphi^{-}\left(P_{1}\right) \cup \cdots \cup \varphi^{-}\left(P_{n_{\max }}\right)\right| \leq\left|\bigcup_{s_{1} \in J_{1}} \varphi^{-}\left(P_{s_{1}}\right)\right|+\cdots+\left|\bigcup_{s_{t} \in J_{t}} \varphi^{-}\left(P_{s_{t}}\right)\right| .
$$

Let us interpret the summands in the right-hand side of (4.2). For example, let us consider the summand $S_{1}:=\left|\bigcup_{s_{1} \in J_{1}} \varphi^{-}\left(P_{s_{1}}\right)\right|$. If $J_{1} \neq \emptyset$, then the points $P_{s_{1}}$ $\left(s_{1} \in J_{1}\right)$ together generate exactly $S_{1}$ CS for $U$ and $S_{1} \leq \sum_{s_{1} \in J_{1}} k_{s_{1}} \leq 2\left|J_{1}\right|$. If $J_{1}=\emptyset$, then $\bigcup_{s_{1} \in J_{1}} \varphi^{-}\left(P_{s_{1}}\right)=\emptyset$ and thus the inequality $S_{1} \leq 2\left|J_{1}\right|$ is valid also. The other summands in the right-hand side of (4.2) are interpreted similarly.

Example 2. Let us consider the Morse potential $U$ depicted in Figure 1. Then, $\mathcal{C}=\left\{\mathcal{E}_{1}, \mathcal{E}_{2}, \mathcal{E}_{3}, \mathcal{E}_{4}\right\}$ and $Y=\left\{P_{1}, P_{2}, P_{3}, P_{4}\right\}$, where $P_{1}=\left(a_{0}, U\left(a_{0}\right)\right), P_{2}=$ $\left(b_{1}, U\left(b_{1}\right)\right), P_{3}=\left(b_{2}, U\left(b_{2}\right)\right)$, and $P_{4}=\left(c_{2}, U\left(c_{2}\right)\right)$. For the multivalued mapping $\varphi: \mathcal{C} \rightrightarrows Y$ defined above, we have $\varphi\left(\mathcal{E}_{1}\right)=\left\{P_{2}\right\}, \varphi\left(\mathcal{E}_{2}\right)=\left\{P_{3}\right\}, \varphi\left(\mathcal{E}_{3}\right)=$ $\left\{P_{3}, P_{4}\right\}, \varphi\left(\mathcal{E}_{4}\right)=\left\{P_{4}\right\}$ and $\varphi^{-}\left(P_{1}\right)=\emptyset, \varphi^{-}\left(P_{2}\right)=\left\{\mathcal{E}_{1}\right\}, \varphi^{-}\left(P_{3}\right)=\left\{\mathcal{E}_{2}, \mathcal{E}_{3}\right\}$, $\varphi^{-}\left(P_{4}\right)=\left\{\mathcal{E}_{3}, \mathcal{E}_{4}\right\}$. Consider the partition $J_{1}:=\{1,2\}, J_{2}:=\{3,4\}$ of the set $\{1,2,3,4\}$. Since $\varphi^{-}\left(P_{1}\right) \cup \varphi^{-}\left(P_{2}\right)=\left\{\mathcal{E}_{1}\right\}$ and $\varphi^{-}\left(P_{3}\right) \cup \varphi^{-}\left(P_{4}\right)=\left\{\mathcal{E}_{2}, \mathcal{E}_{3}, \mathcal{E}_{4}\right\}$, we see that the points $P_{1}$ and $P_{2}$ together generate exactly one CS for $U$ and the points $P_{3}$ and $P_{4}$ together generate exactly three CS for $U$.

Let us prove Theorem 1.

Proof. Let $U$ be a Morse potential.

Case 1. Suppose that (B1) holds; then, $n \geq 3$. It follows from $\lim _{x \rightarrow \pm \infty} U(x)=$ $+\infty$ that $n=2 m+1(m \geq 1), n_{\min }=m+1$, and $n_{\max }=m$. The local maximum points of $U$ are $\xi_{2 i}(1 \leq i \leq m)$. Consider the set $Y=\left\{P_{1}, \ldots, P_{m}\right\}$, where $P_{i}:=\left(\xi_{2 i}, U\left(\xi_{2 i}\right)\right)(1 \leq i \leq m)$, and the partition $J_{i}:=\{i\}(1 \leq i \leq m)$ of the set $\{1, \ldots, m\}$. Since $\left|\varphi^{-}\left(P_{i}\right)\right| \leq 2(1 \leq i \leq m)$, it follows from (4.2) that $N_{C S} \leq 2 m$. Taking into account (3.2), we obtain

$$
N_{N G, N T}=N_{C S}-n_{\min } \leq 2 m-(m+1)=m-1=\lfloor n / 2\rfloor-1 .
$$

Case 2. Suppose that (B2) holds; then, $n \geq 4$. It follows from $\lim _{x \rightarrow \mp \infty} U(x)=$ $\mp \infty$ that $n=2 m(m \geq 2), n_{\min }=m$, and $n_{\max }=m$. The local maximum 
points of $U$ are $\xi_{2 i-1}(1 \leq i \leq m)$. Consider the set $Y=\left\{P_{1}, \ldots, P_{m}\right\}$, where $P_{i}:=\left(\xi_{2 i-1}, U\left(\xi_{2 i-1}\right)\right)(1 \leq i \leq m)$, and the partition $J_{i}:=\{i\}(1 \leq i \leq m)$ of the set $\{1, \ldots, m\}$. Since $\left|\varphi^{-}\left(P_{1}\right)\right|=1,\left|\varphi^{-}\left(P_{s}\right)\right| \leq 2(2 \leq s \leq m)$, it follows from (4.2) that $N_{C S} \leq 1+2(m-1)=2 m-1$. In view of (3.2), we obtain

$$
N_{N G, N T}=N_{C S}-n_{m i n} \leq(2 m-1)-m=m-1=\lfloor n / 2\rfloor-1 .
$$

Case 3. Suppose that (B3) holds. Acting similarly as in Case 2, we obtain $N_{N G, N T} \leq\left\lfloor\frac{n}{2}\right\rfloor-1$.

Case 4. Suppose that (B4) holds; then, $n \geq 5$. It follows from $\lim _{x \rightarrow \pm \infty} U(x)=$ $-\infty$ that $n=2 m+1(m \geq 2), n_{\min }=m$, and $n_{\max }=m+1$. The local maximum points of $U$ are $\xi_{2 j-1}(1 \leq j \leq m+1)$. Consider the set $Y=$ $\left\{P_{1}, \ldots, P_{m+1}\right\}$, where $P_{j}:=\left(\xi_{2 j-1}, U\left(\xi_{2 j-1}\right)\right)(1 \leq j \leq m+1)$. In view of $\lim _{x \rightarrow \pm \infty} U(x)=-\infty$, we conclude that $U$ has a global maximum $\max _{x \in \mathbb{R}} U(x)=: M$. Hence, $M=\max _{j \in K}\left\{U\left(\xi_{2 j-1}\right)\right\}$, where $K:=\{1,2, \ldots, m+1\}$. Suppose that $h$ is the maximum number of $\xi_{2 j-1}, j \in K$, such that $U\left(\xi_{2 j-1}\right)=M$. Then, $h \in\{1, \ldots, m+1\}$ and there is a unique $h$-element subset $H$ of $K$ such that $U\left(\xi_{s}\right)=M$ for every $s \in H$.

Suppose $h=1$. If either $H=\{1\}$ or $H=\{m+1\}$, then consider the partition $J_{1}:=\{1, m+1\}, J_{2}:=K \backslash\{1, m+1\}$ of the set $K$. Since $\left|\bigcup_{s_{1} \in J_{1}} \varphi^{-}\left(P_{s_{1}}\right)\right|=1$ and $\left|\bigcup_{s_{2} \in J_{2}} \varphi^{-}\left(P_{s_{2}}\right)\right| \leq 2\left|J_{2}\right|=2(m-1)$, it follows from (4.2) that $N_{C S} \leq 1+2(m-1)=2 m-1$. In view of (3.2), we obtain

$$
N_{N G, N T}=N_{C S}-n_{\min } \leq(2 m-1)-m=m-1=\lfloor n / 2\rfloor-1 .
$$

If $H=\left\{j_{0}\right\}$, where $j_{0} \in\{2, \ldots, m\}$, then consider the partition $J_{1}:=\left\{j_{0}\right\}$, $J_{2}:=\{1, m+1\}, J_{3}:=K \backslash\left\{1, j_{0}, m+1\right\}$ of the set $K$. Since $\left|\bigcup_{s_{1} \in J_{1}} \varphi^{-}\left(P_{s_{1}}\right)\right|=0$, $\left|\bigcup_{s_{2} \in J_{2}} \varphi^{-}\left(P_{s_{2}}\right)\right|=2$, and $\left|\bigcup_{s_{3} \in J_{3}} \varphi^{-}\left(P_{s_{3}}\right)\right| \leq 2\left|J_{3}\right|=2(m-2)$, it follows from (4.2) that $N_{C S} \leq 0+2+2(m-2)=2 m-2<2 m-1$. In view of (3.2), we obtain

$$
N_{N G, N T}=N_{C S}-n_{\min }<(2 m-1)-m=m-1=\lfloor n / 2\rfloor-1 .
$$

Suppose $h \in\{2, \ldots, m+1\}$. Consider the partition $J_{1}:=H, J_{2}:=K \backslash H$ of the set $K$. Since $\left|\bigcup_{s_{1} \in J_{1}} \varphi^{-}\left(P_{s_{1}}\right)\right|=h-1$ and $\left|\bigcup_{s_{2} \in J_{2}} \varphi^{-}\left(P_{s_{2}}\right)\right| \leq 2\left|J_{2}\right|=2(m+1-$ $h$ ), it follows from (4.2) that $N_{C S} \leq(h-1)+2(m+1-h)=2 m-h+1 \leq 2 m-1$. In view of (3.2), we obtain

$$
N_{N G, N T}=N_{C S}-n_{\min } \leq(2 m-1)-m=m-1=\lfloor n / 2\rfloor-1 .
$$

The proof is complete. 
Corollary 1. Let $U$ be a Morse potential with $n$ critical points. Then, the number of all period annuli for (1.1) satisfies

$$
N \leq \begin{cases}n, & \text { if (B1) is valid; } \\ n-1, & \text { if either (B2) or (B3) is valid; } \\ n-2, & \text { if (B4) is valid }\end{cases}
$$

Proof. It follows from (1.3) and Theorem 1 that

$$
N \leq N_{G}+(\lfloor n / 2\rfloor-1)+n_{m i n}
$$

By the phase plane analysis of (1.2), we have

$$
N_{G}= \begin{cases}1, & \text { if (B1) is valid; } \\ 0, & \text { if either (B2), (B3), or (B4) is valid. }\end{cases}
$$

In view of $(\mathrm{B})$,

$$
n_{\min }= \begin{cases}\lceil n / 2\rceil, & \text { if either }(\mathrm{B} 1),(\mathrm{B} 2), \text { or }(\mathrm{B} 3) \text { is valid } \\ \lceil n / 2\rceil-1, & \text { if }(\mathrm{B} 4) \text { is valid }\end{cases}
$$

Since $\lfloor n / 2\rfloor+\lceil n / 2\rceil=n$ for every integer $n$, see [19, Theorem 2.1.5], it follows from (4.4)-(4.6) that (4.3) is fulfilled.

\section{Proof of the second main theorem}

In a rooted tree, the depth of a node is the length of the unique path connecting the node and the root, see [18, p. 17]. The depth of a rooted tree $T$ is the maximum depth of a node in $T$, see [18, p. 17]; the depth of $T$ is denoted by $\operatorname{depth}(T)$. For a node $u$ of a rooted tree, the number of child nodes for $u$ is denoted by children $(u)$, see [18, p. 18]. If a rooted tree $T$ has $k$ nodes $u_{1}, \ldots, u_{k}$, then, see [18, Lemma 1.44],

$$
\operatorname{children}\left(u_{1}\right)+\cdots+\operatorname{children}\left(u_{k}\right)=k-1 .
$$

In the next proposition, we will indicate the necessary conditions for the Morse potential to ensure equality in (1.4).

Proposition 4. If a Morse potential $U$ with $n$ critical points provides the inequality (1.4) becomes equality, then

(1) $G(U)$ is a binary tree with $k$ nodes,

$$
k=\left\{\begin{array}{cl}
n, & \text { if either (B1), (B2), or (B3) is valid, } \\
n-1, & \text { if (B4) is valid; }
\end{array}\right.
$$

(2) the number of non-root non-leaf nodes in $G(U)$ is $\lfloor n / 2\rfloor-1$;

(3) every non-root non-leaf node in $G(U)$ has two child nodes; 
(4) the number of child nodes of the root $\mathcal{O}$ of $G(U)$ is

$$
\text { children }(\mathcal{O})= \begin{cases}2, & \text { if }(B 1) \text { is valid, } \\ 1, & \text { if either (B2), (B3), or (B4) is valid; }\end{cases}
$$

(5) the equality $\lfloor k / 2\rfloor=\lfloor n / 2\rfloor$ holds, where $k$ is defined by (5.2).

Proof. Let $U$ be a Morse potential with $n$ critical points. It follows from Proposition 2 that $G(U)$ is a rooted tree. Assume $N_{N G, N T}=\left\lfloor\frac{n}{2}\right\rfloor-1$. Let $s$ stand for $\left\lfloor\frac{n}{2}\right\rfloor-1$. In view of Proposition 3, the number of non-root non-leaf nodes in $G(U)$ is $s$ and the number of leaf nodes in $G(U)$ is $n_{\min }$.

Suppose $n=3$; then, $s=0$. Since $n=3$, it follows from (B) that (B1) holds and thus $\lim _{x \rightarrow \pm \infty} U(x)=+\infty$. Hence, the rooted tree $G(U)$ has three nodes: the root and two leaf nodes. Therefore, the statements (1)-(5) are fulfilled.

Suppose $n \geq 4$; then, $s \geq 1$. Let $u_{1}, \ldots, u_{s}$ be non-root non-leaf nodes of $G(U)$ and $k_{j}:=\operatorname{children}\left(u_{j}\right)(1 \leq j \leq s)$. Taking into account Proposition $1(\mathrm{c})$, every non-root non-leaf node of $G(U)$ has at least two child nodes. Hence, $k_{j} \geq 2(1 \leq j \leq s)$. Let $\rho$ stand for children $(\mathcal{O})$. Then, $\rho \geq 1$. Assume that $G(U)$ has $k$ nodes. By (5.1), we obtain

$$
\rho+k_{1}+\cdots+k_{s}=k-1 .
$$

Since $k_{1}+\cdots+k_{s} \geq 2 s$, it follows from (5.4) that

$$
\rho \leq k-1-2 s \text {. }
$$

Suppose that (B1) holds; then, $n=2 m+1(m \geq 2), n_{\min }=m+1$, and $n_{\text {max }}=m$. In view of $\lim _{x \rightarrow \pm \infty} U(x)=+\infty$, we have $\rho \geq 2$. Since $G(U)$ has $s=\left\lfloor\frac{n}{2}\right\rfloor-1=m-1$ non-root non-leaf nodes and $m+1$ leaf nodes, we have $k=1+(m-1)+(m+1)=n$. In accordance with (5.5), we obtain $\rho \leq 2$ and thus $\rho=2$. It follows from (5.4) that

$$
k_{1}+\cdots+k_{s}=2 s
$$

Suppose that either (B2) or (B3) hold; then, $n=2 m(m \geq 2), n_{\min }=m$, and $n_{\max }=m$. Since $G(U)$ has $s=\left\lfloor\frac{n}{2}\right\rfloor-1=m-1$ non-root non-leaf nodes and $m$ leaf nodes, we have $k=1+(m-1)+m=n$. In accordance with (5.5), we obtain $\rho \leq 1$ and thus $\rho=1$. Then, (5.6) is fulfilled.

Suppose that (B4) holds; then $n=2 m+1(m \geq 2), n_{\min }=m$, and $n_{\max }=m+1$. Since $G(U)$ has $s=\left\lfloor\frac{n}{2}\right\rfloor-1=m-1$ non-root non-leaf nodes and $m$ leaf nodes, we have $k=1+(m-1)+m=n-1$. In accordance with (5.5), we obtain $\rho \leq 1$ and thus $\rho=1$. We arrive at the same conclusion that (5.6) is valid.

Thereby, (5.3) and (5.6) are fulfilled. On account of $k_{j} \geq 2(1 \leq j \leq s)$, it follows from $(5.6)$ that $k_{j}=2(1 \leq j \leq s)$ and thus the statements (1)-(4) are valid.

If either (B1), (B2), or (B3) holds, then obviously $\left\lfloor\frac{k}{2}\right\rfloor=\left\lfloor\frac{n}{2}\right\rfloor$ for $k$ defined by (5.2). If (B4) holds, then $n$ is odd and, for $k$ defined by (5.2), we have $\left\lfloor\frac{k}{2}\right\rfloor=\left\lfloor\frac{n-1}{2}\right\rfloor=\left\lfloor\frac{n}{2}\right\rfloor$. Hence, the statement (5) is valid. 
The following corollary indicates the necessary and sufficient conditions providing equality in (1.4).

Corollary 2. Let $U$ be a Morse potential with $n$ critical points. The following assertions are equivalent.

(a) The inequality (1.4) becomes equality.

(b) The rooted tree $G(U)$ is a binary tree with $k$ nodes, where $k$ is defined by (5.2).

(c) The rooted tree $G(U)$ has $k$ nodes, where $k$ is defined by (5.2).

Proof. The assertion (b) is a consequence of (a) in view of Proposition 4 . The assertion (c) is a trivial consequence of (b). Let us prove that (c) implies (a). Assume that the rooted tree $G(U)$ has $k$ nodes, where $k$ is defined by (5.2). On account of Proposition 3, we have

$$
k=N_{N G, N T}+n_{\min }+1 .
$$

Suppose that either (B1), (B2), or (B3) hold; then, in view of (5.2) and (5.7), we obtain $N_{N G, N T}=n_{\max }-1=\left\lfloor\frac{n}{2}\right\rfloor-1$. Suppose that (B4) holds; then, in view of (5.2) and (5.7), we obtain $N_{N G, N T}=n_{\max }-2=\left\lfloor\frac{n}{2}\right\rfloor-1$. Thereby, the inequality (1.4) becomes equality.

Remark 2. The condition that the rooted tree $G(U)$ associated with a Morse potential $U$ is a binary tree is necessary but not sufficient for the inequality (1.4) to become the equality.

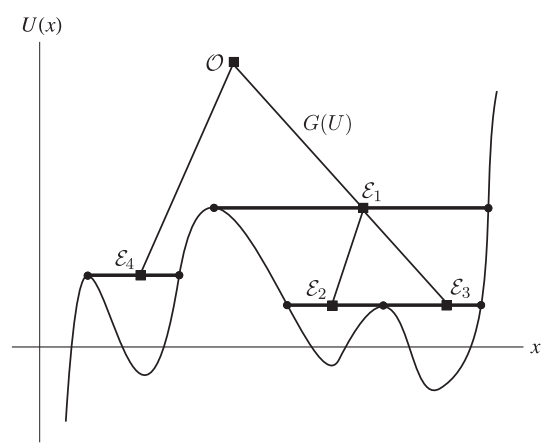

Figure 2. A Morse potential $U$ has six critical points and satisfies (B2). The function $U$ has four $\operatorname{CS} \mathcal{E}_{1}, \mathcal{E}_{2}, \mathcal{E}_{3}$, and $\mathcal{E}_{4}$, which are nodes of the associated rooted tree $G(U)$.

For example, the Morse potential $U$ depicted in Figure 2 has $n=6$ critical points and satisfies (B2); the associated rooted tree $G(U)$ is a binary tree with $k=5$ nodes. Since (5.2) is not fulfilled, it follows from Corollary 2 that the inequality (1.4) is strict.

Let $k$ be an integer greater than one and let $s$ stand for $\left\lfloor\frac{k}{2}\right\rfloor-1$. Based on Proposition 4 , to every $k$, we associate a binary tree $T_{k}$ as follows. Consider 
a binary tree $T_{s+2}^{\prime}$ with $s+2$ nodes and the depth $s+1$. Then, $T_{s+2}^{\prime}$ has $s$ non-root non-leaf nodes and one leaf node. Add a leaf node to each non-root non-leaf node of $T_{s+2}^{\prime}$ and add a leaf node to the root of $T_{s+2}^{\prime}$ if $k$ is odd. The binary tree $T_{k}$ obtained has $k$ nodes, $\left\lfloor\frac{k}{2}\right\rfloor-1$ non-root non-leaf nodes, and $\left\lceil\frac{k}{2}\right\rceil$ leaf nodes, see Figure 3. We see that $\operatorname{depth}\left(T_{k}\right)=\operatorname{depth}\left(T_{s+2}^{\prime}\right)=\left\lfloor\frac{k}{2}\right\rfloor$ and every non-root non-leaf node in $T_{k}$ has two child nodes. Among binary trees with $k$ nodes, $T_{k}$ provides an example of a binary tree that has the minimum number $\left\lfloor\frac{k}{2}\right\rfloor$ of non-leaf nodes, see $\left[19\right.$, p. 216], and the maximum number $\left\lceil\frac{k}{2}\right\rceil$ of leaf nodes, see [19, p. 217].

Let us prove Theorem 2 .

Proof. The idea of proof is to define a Morse potential $U$ with $n$ critical points in such a way that the associated rooted tree $G(U)$ is a binary tree $T_{k}$, where $k$ is defined by (5.2). Taking into account Corollary 2 and the properties of $T_{k}$ described above, the Morse potential $U$ will provide the maximum number $\left\lfloor\frac{n}{2}\right\rfloor-1$ of nonglobal nontrivial period annuli for (1.1),

$$
N_{N G, N T}=\lfloor n / 2\rfloor-1 .
$$

Consider functions $p_{\sigma}(x):=\sigma(x-\sin 2 x)$,

$h_{\sigma, a}(\alpha ; x):=a\left(x^{3}-\alpha^{3}\right)-3 a x \alpha(x-\alpha)+\sigma x-2 \sigma(x-\alpha) \cos (2 \alpha)-\sigma \sin (2 \alpha)$,

where $\sigma, a \in\{-1,1\}$ and $\alpha \in \mathbb{R}$ are parameters. Next, we define three Morse potentials $U_{1}, U_{2}$, and $U_{3}$ with $n$ critical points $C^{2}$-smoothly gluing $p_{\sigma}(x)$ and two copies of $h_{\sigma, a}(\alpha ; x)$ at some inflections points $\frac{\pi n}{2}(n \in \mathbb{Z})$ of $p_{\sigma}(x)$ in such a way that $U_{1}, U_{2}$, and $U_{3}$ satisfy (B1), (B2), and (B3), respectively, and $G\left(U_{i}\right)=T_{n}, i \in\{1,2,3\}$. Then, it follows from the above discussion that (5.8) is fulfilled.

Case 1. Suppose $n=2 m+1$, where $m \geq 1$. The function

$$
U_{1}(x):=\left\{\begin{array}{cl}
h_{1,-1}(0 ; x), & \text { if } \quad x \leq 0 \\
p_{1}(x), & \text { if } \quad 0 \leq x \leq \frac{\pi n}{2}, \\
h_{1,1}\left(\frac{\pi n}{2} ; x\right), & \text { if } \quad x \geq \frac{\pi n}{2}
\end{array}\right.
$$

has $n$ critical points and satisfies (A) and (B1). We have $n_{\min }=m+1$ and $n_{\max }=m$. The function $U_{1}$ has the local maxima $U_{1}\left(-\frac{\pi}{6}+\pi j\right)=\frac{\sqrt{3}}{2}-\frac{\pi}{6}+\pi j$ $(1 \leq j \leq m)$. Since $U_{1}\left(-\frac{\pi}{6}+\pi\right)<\cdots<U_{1}\left(-\frac{\pi}{6}+\pi m\right)$, we see that $G\left(U_{1}\right)=$ $T_{n}$ and thus (5.8) is fulfilled. (The graph of the function $U_{1}$ if $n=11$ is depicted in Figure 4 (a); the rooted tree $G\left(U_{1}\right)=T_{11}$ if $n=11$ is depicted in Figure 3.)

Case 2. Suppose $n=2 m$, where $m \geq 2$. The function

$$
U_{2}(x):=\left\{\begin{array}{cl}
h_{-1,1}(0 ; x), & \text { if } \quad x \leq 0, \\
p_{-1}(x), & \text { if } \quad 0 \leq x \leq \frac{\pi n}{2}, \\
h_{-1,1}\left(\frac{\pi n}{2} ; x\right), & \text { if } \quad x \geq \frac{\pi n}{2},
\end{array}\right.
$$

has $n$ critical points and satisfies (A) and(B2). We have $n_{\min }=m$ and $n_{\max }=$ $m$. The function $U_{2}$ has the local maxima $U_{2}\left(\frac{\pi}{6}+\pi j\right)=\frac{\sqrt{3}}{2}-\frac{\pi}{6}-\pi j(0 \leq$ 


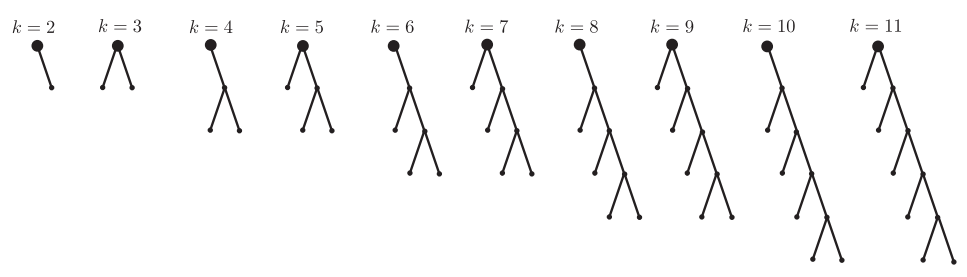

Figure 3. Binary trees $T_{k}(2 \leq k \leq 11)$.

$j \leq m-1)$. Since $U_{2}\left(\frac{\pi}{6}\right)>\cdots>U_{2}\left(\frac{\pi}{6}+\pi(m-1)\right)$, we see that $G\left(U_{2}\right)=T_{n}$ and thus (5.8) is fulfilled. (The graph of the function $U_{2}$ if $n=10$ is depicted in Figure $4(\mathrm{~b})$; the rooted tree $G\left(U_{2}\right)=T_{10}$ if $n=10$ is depicted in Figure 3.)

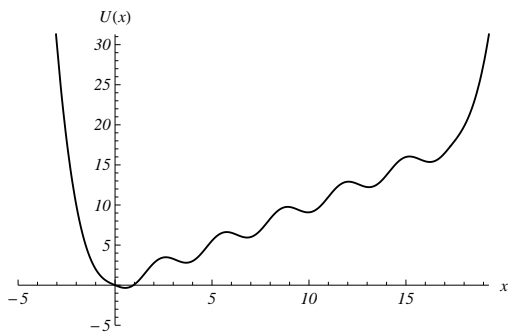

(a) The graph of the function $U_{1}$ defined by (5.9) if $n=11$.

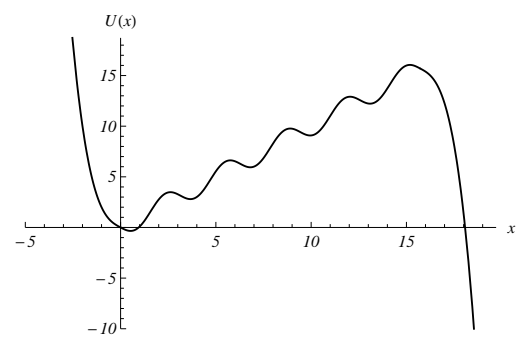

(c) The graph of the function $U_{3}:=-U_{2}$, where $U_{2}$ is defined by (5.10), if $n=$

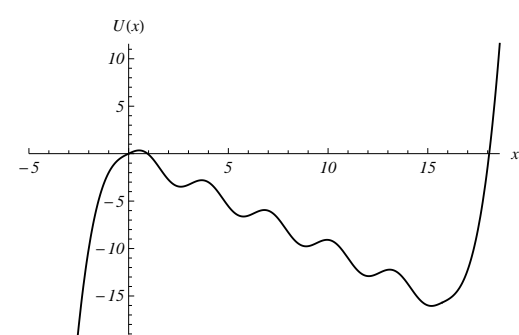

(b) The graph of the function $U_{2}$ defined by (5.10) if $n=10$.

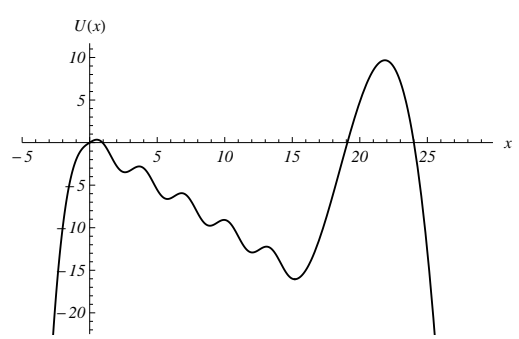

(d) The graph of the function $U_{4}$ defined by (5.11) if $n=11$. 10.

Figure 4. Illustration to the proof of Theorem 2.

Case 3. Suppose $n=2 m$, where $m \geq 2$. The function $U_{3}:=-U_{2}$, where $U_{2}$ is defined by (5.10), has $n$ critical points and satisfies (A) and (B3). By the similar arguments as in Case 2, we conclude that (5.8) is fulfilled. (The graph of the function $U_{3}$ if $n=10$ is depicted in Figure 4 (c); the rooted tree $G\left(U_{3}\right)=T_{10}$ if $n=10$ is depicted in Figure 3.)

Case 4. Suppose $n=2 m+1$, where $m \geq 2$. Consider a function

$$
\begin{aligned}
g_{b}(\beta ; x):= & b\left(x^{3}-\beta^{3}\right)-3 b x \beta(x-\beta)-\left(2 x^{2}-4 x \beta+2 \beta^{2}-1\right) \sin (2 \beta) \\
& +2(x-\beta) \cos (2 \beta)-x
\end{aligned}
$$


where $b$ and $\beta$ are real parameters. The function

$$
U_{4}(x):=\left\{\begin{array}{cl}
h_{-1,1}(0 ; x), & \text { if } \quad x \leq 0, \\
p_{-1}(x), & \text { if } \quad 0 \leq x \leq-\frac{\pi}{6}+\pi m, \\
g_{b}\left(-\frac{\pi}{6}+\pi m ; x\right), & \text { if } \quad x \geq-\frac{\pi}{6}+\pi m,
\end{array}\right.
$$

where $b:=-\frac{1}{\sqrt{2+2 \pi m}}, C^{2}$-smoothly glues together the three functions at the inflection point $x=0$ and the local minimum point $\beta=-\frac{\pi}{6}+\pi m$ of $p_{-1}(x)$ in such a way that $U_{4}$ has $n$ critical points and satisfies (A) and (B4). We have $n_{\min }=m$ and $n_{\max }=m+1$. The function $U_{4}$ has the local maxima $U_{4}\left(\frac{\pi}{6}+\pi j\right)=\frac{\sqrt{3}}{2}-\frac{\pi}{6}-\pi j \quad(0 \leq j \leq m-1)$ and $U_{4}(\delta)=\frac{4 \sqrt{3}}{9 b^{2}}-\frac{\sqrt{3}}{2}+\frac{\pi}{6}-\pi m$, where $\delta=\pi m-\frac{\pi}{6}-\frac{2 \sqrt{3}}{3 b}>\frac{\pi}{6}+\pi(m-1)$. Since

$$
U_{4}(\delta)>1>U_{4}\left(\frac{\pi}{6}\right)>0>U\left(\frac{\pi}{6}+\pi\right)>\cdots>U\left(\frac{\pi}{6}+\pi(m-1)\right),
$$

we see that $G\left(U_{4}\right)=T_{n-1}$ and thus (5.8) is fulfilled. (The graph of the function $U_{4}$ if $n=11$ is depicted in Figure $4(\mathrm{~d})$; the rooted tree $G\left(U_{4}\right)=T_{10}$ if $n=11$ is depicted in Figure 3.)

The proof is complete.

Corollary 3. Let $U$ be a Morse potential with $n$ critical points. The maximum number of all period annuli for (1.1) is equal to $n$ if (B1) is valid, $n-1$ if either (B2) or (B3) is valid, and $n-2$ if (B4) is valid.

Proof. It follows from Theorem 2 and Corollary 1 that the functions $U_{i}$, $i \in\{1,2,3,4\}$, considered in the proof of Theorem 2 , provide the maximum number of all period annuli for (1.1) indicated in the statement.

Remark 3. There exist Morse potentials $U$ with $n$ critical points such that the inequality (1.4) becomes equality, and the rooted tree $G(U)$ is a binary tree different from $T_{k}$, where $k$ is defined by (5.2).

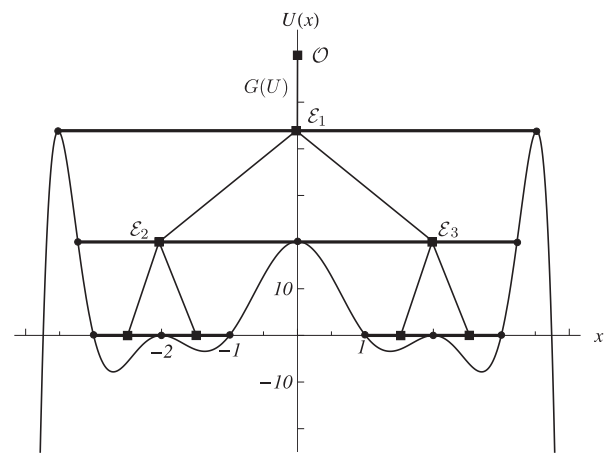

Figure 5. A Morse potential $U(x)=\frac{1}{100}\left(14-x^{2}\right)\left(x^{2}-9\right)\left(x^{2}-4\right)^{2}\left(x^{2}-1\right)$ and the associated rooted tree $G(U)$. 
For example, let us consider a Morse potential $U(x)=\frac{1}{100}\left(14-x^{2}\right)\left(x^{2}-\right.$ $9)\left(x^{2}-4\right)^{2}\left(x^{2}-1\right)$, which has $n=9$ critical points and satisfies the condition (B4). The rooted tree $G(U)$, see Figure 5 , is a binary tree different from $T_{8}$ since $\operatorname{depth}(G(U))=3$ and $\operatorname{depth}\left(T_{8}\right)=4$. The binary tree $G(U)$ has three non-root non-leaf nodes $\mathcal{E}_{1}, \mathcal{E}_{2}$, and $\mathcal{E}_{3}$ and consequently the inequality (1.4) becomes equality.

\section{Conclusions}

In our paper, we consider a second order scalar differential equation with a Morse potential. We present the upper bound for the number of nonglobal nontrivial period annuli for the equation and, as a consequence, the upper bound for the number of all period annuli for the equation. We prove that these bounds are sharp, indicating examples of Morse potentials. In our reasoning, we assign a rooted tree to each Morse potential. The associated with Morse potentials rooted trees have at least three nodes, and every non-root non-leaf node, if any exists, has at least two child nodes. In further research, it would be interesting (a) to describe the Morse potentials with a given rooted tree, (b) to investigate the maximum number of period annuli for potentials with a finite limit at minus or plus infinity.

\section{Acknowledgement}

The authors are grateful to the anonymous referees for careful reading and valuable comments that helped to improve this article.

\section{References}

[1] V.I. Arnold. Ordinary Differential Equations. Springer, Berlin, 1992.

[2] S. Atslega and F. Sadyrbaev. On periodic solutions of Liénard type equations. Mathematical Modelling and Analysis, 18(5):708-716, 2013. https://doi.org/10.3846/13926292.2013.871651.

[3] C. Berge. Topological Spaces: Including a Treatment of Multi-Valued Functions, Vector Spaces and Convexity. Dover Publications, Inc., Mineola, NY, 1997.

[4] S. Biasotti, D. Giorgi, M. Spagnuolo and B. Falcidieno. Reeb graphs for shape analysis and applications. Theoretical Computer Science, 392(1):5-22, 2008. https://doi.org/10.1016/j.tcs.2007.10.018.

[5] C. Christopher and C. Li. Limit Cycles of Differential Equations. Advanced Courses in Mathematics. CRM Barcelona, Birkhäuser Verlag, Basel, 2007.

[6] F. Dumortier. Limit cycles of differential equations (book review of MR2325099). Bulletin of the American Mathematical Society, 46(4):697-701, 2009. https://doi.org/10.1090/S0273-0979-09-01267-1.

[7] I. Gelbukh. Loops in Reeb graphs of $n$-manifolds. Discrete $\& 3$ Computational Geometry, 59(4):843-863, 2018. https://doi.org/10.1007/s00454-017-9957-9.

[8] Y. Kozmina. On the polynomials of optimal shape generating maximum number of period annuli. Innovative Infotechnologies for Science, Business and Education, 2(13):13-19, 2012. Available from Internet: http://old.kolegija.lt/ dokumentai_img/IITSBE2012213.pdf. 
[9] Y. Kozmina and F. Sadyrbaev. On a maximal number of period annuli. Abstract and Applied Analysis, 2011:8, 2011. https://doi.org/10.1155/2011/393875.

[10] F. Mańosas and J. Villadelprat. A note on the critical periods of potential systems. International Journal of Bifurcation and Chaos, 16(3):765-774, 2006. https://doi.org/10.1142/S0218127406015155.

[11] P. Mardešíc, D. Marín and J. Villadelprat. The period function of reversible quadratic centers. Journal of Differential Equations, 224(1):120-171, 2006. https://doi.org/10.1016/j.jde.2005.07.024.

[12] W. Marzantowicz, M. Kaluba and N. Silva. On representation of the Reeb graph as a sub-complex of manifold. Topological Methods in Nonlinear Analysis, 45(1):287-307, 2015. https://doi.org/10.12775/TMNA.2015.015.

[13] Ł.P. Michalak. Realization of a graph as the Reeb graph of a Morse function on a manifold. Topological Methods in Nonlinear Analysis, 52(2):749-762, 2018. https://doi.org/10.12775/TMNA.2018.029.

[14] L. Nicolaescu. An Invitation to Morse Theory. Universitext, Springer, New York, 2007. https://doi.org/10.1007/978-0-387-49510-1.

[15] M. Potter. Set Theory and its Philosophy: A Critical Introduction. Oxford University Press, New York, 2004. https://doi.org/10.1093/acprof:oso/9780199269730.001.0001.

[16] D. Rojas. On the upper bound of the criticality of potential systems at the outer boundary using the Roussarie-Ecalle compensator. Journal of Differential Equations, 267(6):3922-3951, 2019. https://doi.org/10.1016/j.jde.2019.04.021.

[17] M. Sabatini. Liénard limit cycles enclosing period annuli, or enclosed by period annuli. Rocky Mountain Journal of Mathematics, 35(1):253-266, 2005. https://doi.org/10.1216/rmjm/1181069780.

[18] G. Valiente. Algorithms on Trees and Graphs. Springer-Verlag, Berlin, 2002. https://doi.org/10.1007/978-3-662-04921-1.

[19] D. Vrajitoru and W. Knight. Practical Analysis of Algorithms. Springer, Cham, 2014. https://doi.org/10.1007/978-3-319-09888-3.

[20] D.B. West. Introduction to Graph Theory. Prentice Hall, Inc., Upper Saddle River, NJ, 1996.

[21] L. Yang and X. Zeng. The period function of potential systems of polynomials with real zeros. Bulletin des Sciences Mathématiques, 133(6):555-577, 2009. https://doi.org/10.1016/j.bulsci.2009.05.002.

[22] P. Yu. Chapter 1 bifurcation, limit cycle and chaos of nonlinear dynamical systems. In J.Q. Sun and A.C.J. Luo(Eds.), Bifurcation and Chaos in Complex Systems, volume 1 of Edited Series on Advances in Nonlinear Science and Complexity, pp. 1-125. Elsevier Science, 2006. https://doi.org/10.1016/S15746909(06)01001-X. 